\title{
RISK OF BLOOD EXPOSURES AMONG HOME HEALTHCARE WORKERS: LINKING QUALITATIVE AND QUANTITATIVE METHODS
}

Margaret Quinn, ${ }^{1}$ Pia Markkanen, ${ }^{1}$ Catherine Galligan, ${ }^{1}$ Hyun Kim, ${ }^{2}$ Rebecca Gore, ${ }^{1}$ Susan Sama, ${ }^{1}$ David Kriebel, ${ }^{1}$ Angela Laramie, ${ }^{3}$ Letitia Davis ${ }^{3}{ }^{1}$ University of Massachusetts, Lowell, USA; ${ }^{2}$ Hofstra University, Hempstead, USA; ${ }^{3}$ Massachusetts Department of Public Health, Boston, USA

10.1136/oemed-2011-100382.105

Objectives This study quantitatively assessed risks of blood exposures among home healthcare (HHC) nurses and aides. HHC workers are at risk of bloodborne pathogen exposures from needlesticks and injuries from sharp medical devices ("sharps") and contaminated blood and body fluids. Risk estimates of sharps injuries (SI) and other blood and body fluid exposures (BBFE) exist for hospitals, but little is known of HHC risks.

Methods Focus groups (FGs) of HHC workers and interviews with HHC managers and union representatives were conducted followed by a survey among HHC workers at 26 worksites and 2 labor unions.

Results 1225 questionnaires were completed. Over their entire HHC career, $34.9 \%$ of nurses and $6.4 \%$ of aides had at least one SI; $15.1 \%$ of nurses and $6.7 \%$ of aides had at least one other BBFE. Annual SI rates per 100 full-time equivalent (FTE) employees were 5.1 SI/100 FTE for nurses, 1.0 SI/100 FTE for aides. Nurses working on a contingency basis had the highest rate of SI (13.4/100 FTE); full-time nurses had the lowest (2.9/100 FTE). FGs and interviews contributed to all aspects of exposure assessment methods and found that most HHC workers liked their jobs, citing more autonomy and ability to establish caring relationships with patients relative to hospital work.

Conclusions Interventions are needed to prevent SI and BBFE in HHC. FGs and interviews combined with surveys provided a richer understanding of risks and opportunities for prevention. As interventions are developed, we need to enhance job dimensions that make dignified, meaningful care possible. 